\title{
THE POSITIVE CONE IN BANACH ALGEBRAS
}

\author{
BY \\ J. L. KELLEY(1) AND R. L. VAUGHT(2)
}

This paper concerns Banach algebras which are real or ${ }^{*}$ algebras and possess a unit. The principal method of attack is via an ordering of the algebra, the positive cone being the closure of the set of sums of squares (sums of elements $x x^{*}$ ) in contrast to the positive open cone used by Raikov [9] $\left(^{3}\right)$ and others. An important role is played by an identity on norms, which together with a few preliminary lemmas is proved in $\$ 1$. In $\$ 2$ the real homomorphisms of a real commutative algebra are found to be the extreme points of the intersection of the dual cone and the unit sphere in the adjoint of the algebra, and the "real radical" is shown to consist of elements $x$ such that $-x^{2}$ is approximately a sum of squares. The theorem of Arens [1] characterizing real function algebras is derived. In $\S \S 3$ and 4 these results are applied to ${ }^{*}$ algebras. The new norm of an element $x$, which Gelfand and Naimark [3] introduced by means of positive functionals, is proved to be the square root of the distance from $-x x^{*}$ to the positive cone. Some results relating general ${ }^{*}$ algebras to operator algebras, including the representation theorem of Gelfand and Naimark [2], are derived. In \$5, a refinement of the basic identity is established for the Fourier transform of a measure (discrete + absolutely continuous) on a locally compact Abelian group.

R. V. Kadison [5] has recently investigated Banach algebras by means of an order relation. The positive cone he uses is identical with that used here only when $1+x x^{*}$ always has an inverse. The principal overlap with Kadison's work, outside of the deduction of certain known theorems by order methods, seems to be the geometric characterization of the real homomorphisms of a real algebra (see 2.1). Like Kadison's work, this paper is essentially self-contained. (Some notable exceptions occur in \$5.)

\section{Preliminaries.}

1.1. Definitions. A set $C$ is a cone in a real Banach space $R$ if it is closed, nonvoid, the sum of two members of $C$ is a member of $C$, and non-negative scalar multiples of members of $C$ are members of $C$. If $C$ is a cone in $R$, then $C^{\prime}$, the dual cone, is the set of bounded linear functionals which are non-

Presented to the International Congress of Mathematicians, September 2, 1951; received by the editors March 22, 1952.

(1) This work was done in part under Contract N7-onr-434, Task order III, Navy Department, Office of Naval Research.

(2) Atomic Energy Commission Predoctoral Fellow, during a part of the preparation of this paper.

$\left.{ }^{8}\right)$ Numbers in brackets refer to the bibliography at the end of this paper. 
negative on $C$, i.e., $\left\{f: f \in R^{*}\right.$ and $f(c) \geqq 0$ for $\left.c \in C\right\}$. (Clearly $C^{\prime}$ is a cone in $R^{*}$.)

1.2. Lemma. If $C$ is a cone in $R$, then $x \in C$ if and only if $f(x) \geqq 0$ for each $f \in C^{\prime}$.

Proof. Suppose $x \notin C$. Then, since $C$ is closed and convex, there is an $f \in R^{*}$ with $f(x)<f(y)$ for all $y \in C$, by the Hahn-Banach theorem( $\left.{ }^{4}\right)$. Thus $f(x)<0=f(0)$, and, considering positive scalar multiples of any $y \in C$, it is clear that $f(y) \geqq 0$, and hence $f \in C^{\prime}$.

1.3. Lemma. Let $C$ be a cone in $R, x$ an element of $R$, and $e$ an element of $C$ such that $\|e\|=1$ and such that $C$ includes $\{y:\|y-e\| \leqq 1\}$. Then:

(a) $\|f\|=f(e)$, for $f \in C^{\prime}$,

(b) dist $(-x, C)=\sup \left\{f(x): f \in C^{\prime}\right.$ and $\left.\|f\| \leqq 1\right\}$, and

(c) the minimum distance in (b) is attained at the point

$$
[\operatorname{dist}(-x, C)] \cdot e-x \text {. }
$$

Proof. Since $\|e\|=1,\|f\| \geqq f(e)$. If $\|y\| \leqq 1$, then by hypothesis $e \pm y \in C$, so that $f(e \pm y) \geqq 0$ and $f(e) \geqq|f(y)|$. Statement (a) is thereby established. If $c \in C, f \in C^{\prime}$, and $\|f\| \leqq 1$, then $\|x+c\| \geqq|f(x+c)| \geqq f(x)+f(c) \geqq f(x)$, which establishes one inequality for (b). Let $r=\sup \left\{f(x): f \in C^{\prime}\right.$ and $\left.\|f\| \leqq 1\right\}$. Then if $f \in C^{\prime}$ and $f(e)=1, f(r e-x)=r-f(x) \geqq 0$, and if $f(e)=0$, then $\|f\|=0$ and $f(r e-x)=0$; in any case $f(r e-x) \geqq 0$ for each $f \in C^{\prime}$. Hence, by $1.2, r e-x \in C$, and dist $(-x, C)=\inf \{\|x+c\|: c \in C\}=\|x+r e-x\|=r$. This proves the lemma.

1.4. Remarks. Under the hypotheses of this lemma; any linear functional $f$ which is non-negative on $C$ is necessarily bounded (by $f(e)$ ). For $C$ a cone in $R$ let $\Sigma=\left\{f: f \in C^{\prime}\right.$ and $\left.\|f\| \leqq 1\right\}$. Recall that $x$ is an extreme point of a convex set $K$ if $x$ is not an interior point of any line segment lying in $K$, i.e., if whenever $x=t y+(1-t) z$, where $y$ and $z$ belong to $K$ and $0<t<1$, then $y=z$. We now derive a property of the extreme points of $\Sigma$.

1.5. Lemma. Let $C$ be a cone, $e \in C$ such that $\|e\|=1$ and such that $C$ includes $\{x:\|x-e\| \leqq 1\}$, let $\Sigma=\left\{g: g \in C^{\prime}\right.$ and $\left.\|g\| \leqq 1\right\}$, and let $f$ be an extreme point of $\Sigma$. Then for each $g \in \Sigma$ such that $f-g \in C^{\prime}, g=g(e) \cdot f$.

Proof. Let $g \in \Sigma$ and $f-g \in C^{\prime}$. Since $\|f\|=f(e)$, by 1.3 , it is clear that $f(e)$ $=0$ or 1 . If $f(e)=0$, then since $g(e) \geqq 0$ and $f(e)-g(e) \geqq 0$, we have $\|g\|=g(e)$ $=0$, and the conclusion is clear. Suppose $f(e)=1$. If $f=g$ or $g=0$, the conclu-

(4) Cones (not necessarily closed) have been studied by Krein and Rutman [7] and others, and Krein and Rutman established a theorem on the extension of linear functionals non-negative on a cone. Lemmas 1.2 and 1.3 could be derived from their theorem, but follow more easily and directly from the following well known consequence of the familiar Hahn-Banach extension theorem: If $K$ is a closed convex set in a real Banach space $R$, and $x \notin K$, then there is an $f \in R^{*}$ with $f(x)<f(y)$, for all $y \in R$. 
sion is obvious, and otherwise we have

$$
f=g(e)[g / g(e)]+[f(e)-g(e)][(f-g) /(f(e)-g(e))],
$$

and since $f$ is extreme, $f=g / g(e)$, which is the desired conclusion.

1.6. Lemma. Let $K$ be a nonvoid compact convex subset of a real linear topological space and $f$ a continuous linear functional. Then the supremum of $f(x)$ for $x \in K$ is attained at an extreme point of $K$.

Proof. Let $L=\{x: x \in K$ and $f(x)=\sup \{f(y): y \in K\}\}$. Because $K$ is compact, $L$ is nonvoid, and clearly $L$ is compact and convex. If $S$ is a line segment in $K$ such that an interior point belongs to $L$, then since $f$ is linear, $S \subseteq L$. Consequently each extreme point of $L$ is an extreme point of $K$, and, by the Krein-Milman theorem [7], there are extreme points of $L$.

2. Real Banach algebras. Suppose that $R$ is a real Banach algebra with unit 1 such that $\|1\|=1$. A real homomorphism is a linear functional $h$ such that $h(x y)=h(x) h(y)$ for all $x, y \in R$. Let $P$ be the closure in $R$ of the set of all finite sums of squares. Clearly (a) $P$ is a cone in $R$; (b) $1 \in P$; (c) if $R$ is a commutative algebra, $P$ is closed under multiplication; and (d) for $f \in R^{*}$, $f \in P^{\prime}$ if and only if $f\left(x^{2}\right) \geqq 0$ for each $x \in R$. Let $\Sigma$ be the intersection of $P^{\prime}$ with the unit sphere in $R^{*}$.

We observe that since the power series for $(1-t)^{1 / 2}$ converges uniformly and absolutely on $-1 \leqq t \leqq 1$, if $\|1-x\| \leqq 1$, then $x$ has a square root, the root being given by the power series in $1-x$. It follows easily that real homomorphisms are bounded and members of $P^{\prime}$; more generally, since $P$ includes the unit sphere about 1, we may apply Lemmas 1.3 and 1.5.

2.1. THEOREM. If $R$ is commutative, the set of extreme points of $\Sigma$ is identical with the set of real homomorphisms of $R$.

Proof. Let $f$ be an extreme point of $\Sigma$. Since 0 is certainly a real homomorphism of $R$, we may suppose $f \neq 0$ and hence $f(1)=1$. Let $\|x\| \leqq 1$ and $x \in P$ and set $f_{x}(y)=f(x y)$ for $y \in R$. It is easy to check that $f \in \Sigma$. Also $1-x \in P$, and hence if $y \in P$, then $(1-x) y \in P$ and $\left(f-f_{x}\right)(y)=f((1-x) y) \geqq 0$. Thus $f-f_{x} \in P^{\prime}$ and, by $1.5, f_{x}=f_{x}(1) \cdot f$, i.e., $f(x y)=f(x) f(y)$ for all $y \in R$. The restriction on $\|x\|$ is easily removed, and since any $x \in R$ is the difference of two members of $P$ (in fact, $\left.4 x=(1+x)^{2}-(1-x)^{2}\right)$, it follows that $f(x y)$ $=f(x) f(y)$ for all $x, y \in R$.

Conversely, suppose $f$ is a real homomorphism of $R$. Since $f \in P^{\prime},\|f\|$ $=f(1)=f\left(1^{2}\right)=(f(1))^{2}=0$ or 1 and $f \in \Sigma$. Suppose $f=t g+(1-t) h$ where $0<t<1$ and $g, h \in \Sigma$. If $f(1)=0, f=g=0$, so we may assume $f(1)=1$. It is easy to check that $g(x y)$ defines a real inner product on $R$, and consequently, by the Cauchy-Schwartz inequality, $g(x y)^{2} \leqq g\left(x^{2}\right) g\left(y^{2}\right)$. Hence whenever $f(x)=0$, we have $f\left(x^{2}\right)=0$ and consequently, since $g, h \in \Sigma, g\left(x^{2}\right)=0$, and so $g(x)^{2}$ $\leqq g\left(x^{2}\right) g(1)=0$ and thus, finally, $g(x)=0$. In particular $f(x-f(x) \cdot 1)=0$ and 
so $g(x)=g(1) f(x)$ for all $x \in R$. Since $1=f(1)=\operatorname{tg}(1)+(1-t) h(1), 0<t<1$, we have $g(1)=1$ and hence $g=f$. Thus $f$ is extreme.

Let $X_{0}$ be the set of all real homomorphisms of $R$, and let $X=X_{0}-\{0\}$ be the set of nontrivial homomorphisms. If $R^{*}$ is topologized by the $w^{*}$ topology, i.e., the topology of point-wise convergence, then the unit sphere in $R^{*}$ is $w^{*}$ compact, and one easily verifies that $X_{0}$ is a $w^{*}$ closed subset. Since $X$ is the intersection of $X_{0}$ with the $w^{*}$ closed set $\{f: f(1)=1\}$, the set $X$ is $w^{*}$ compact.

There is a natural map $U$ of $R$ into the algebra $R(X)$ of all $w^{*}$ continuous real-valued functions on $X$, defined by

$$
U(x)(f)=f(x), \quad \text { for } x \in R, f \in X .
$$

We assume $R(X)$ has the usual supremum norm. Because $X$ consists of homomorphisms, $U$ is a homomorphism. We summarize the properties of $U$ :

2.2. TheOREM. Let $R$ be a commutative real Banach algebra with unit of norm 1 , let $P$ be the closure of the set of all sums of squares, let $X$ be the set of nontrivial real homomorphisms $\left(^{(5)}\right.$ with the $w^{*}$ topology, and let $U$ be the natural map of $R$ into the real continuous functions $R(X)$ on $X$. Then

(a) $U$ maps $R$ onto a dense subalgebra of $R(X)$,

(b) if $x \in R$, dist $(-x, P)=\max [0$, sup $\{f(x): f \in X\}]$, and

(c) in particular,

$$
\|U(x)\|=\max [\operatorname{dist}(-x, P), \operatorname{dist}(x, P)]=\operatorname{dist}\left(-x^{2}, P\right)^{1 / 2} .
$$

Proof. It is clear that if $f \neq g$ and $f, g \in X$, then, for some $x \in R, f(x)$ $=U(x)(f) \neq U(x)(g)$. Consequently the image of $R$ in $R(X)$ distinguishes points of $X$ and, by the Stone-Weierstrass approximation theorem [13], must be dense in $R(X)$. To prove (b), first observe that $P^{\prime}$ is $w^{*}$ closed. Since $\Sigma$ is $w^{*}$ compact, Lemma 1.6 shows that sup $\{f(x): f \in \Sigma\}$ is attained for an extreme point of $\Sigma$. But, by $2.1, X_{0}$ is the set of these extreme points, so, by 1.3

$$
\operatorname{dist}(-x, P)=\sup \left\{f(x): f \in X_{0}\right\}=\max [0, \sup \{f(x): f \in X\}] .
$$

The norm equality is straightforward.

2.3. REMARKs. The real radical of a commutative Banach algebra with unit is the kernel of the map $U$. From the preceding results it is clear that the following statements are equivalent:

(a) $x$ belongs to the radical,

( $\left.{ }^{\sharp}\right)$ There may be no real homomorphisms of $R$ except 0 (as in the case where $R$ is the complex numbers.) The meaning of Theorem 2.2 may not be quite "clear" in this case. For the sake of completeness, therefore, we remark that 0 is the only real homomorphism of $R$ if and only if $R=P$. To make 2.2 intelligible in this case we must define $\|U(x)\|=\max [0, \sup \{f(x): f \in X\}]$, since $X$ may be empty. The proof of 2.4 then contains an assurance that $X$ is not empty under the additional hypotheses there. The situation in 3.4, in the case where $Y$ is empty, is analogous. 
(b) both $x$ and $-x$ may be approximated by sums of squares,

(c) $f(x)=0$ for every real homomorphism $f$,

(d) $-x^{2}$ can be approximated by a sum of squares.

Thus there are "sufficiently many" real homomorphisms, i.e., the radical is $\{0\}$ if and only if 0 is an extreme point of the positive cone. It is of particular interest to know conditions under which the natural map of $R$ into $R(X)$ is an isometry. We now prove the theorem of Arens [1].

2.4. THEOREM. If $R$ is a real commutative Banach algebra with unit such that $\left\|x^{2}\right\|=\|x\|^{2}$ and $\left\|x^{2}+y^{2}\right\| \geqq\left\|x^{2}\right\|$ for each $x, y \in R$, then $R$ is isomorphic and isometric to the Banach algebra of all real-valued continuous functions on the space of all nontrivial real homomorphisms of $R$.

Proof. Observe that if $\|x\| \leqq 1$ and $\|1-x\| \leqq 1$, then both $x$ and $1-x$ are squares. Conversely if $x=u^{2}$ and $1-x=v^{2}$, then $1=u^{2}+v^{2}$ and, in view of the hypothesis of the theorem $\|x\|$ and $\|1-x\|$ are less than or equal to 1 . Now suppose $x$ and $y$ are squares and also $\|x\|,\|y\| \leqq 1$. Then $1-x$ and $1-y$ are squares and so $\|1-x\|,\|1-y\| \leqq 1$. Hence $\|1-(x+y) / 2\| \leqq 1 / 2(\|1-x\|$ $+\|1-y\|) \leqq 1$, and so $(x+y) / 2$ and hence $x+y$ are squares. It follows easily that the sum of any two squares is a square. But then squares are dense in $P$ and so, for any $x \in R,\|U(x)\|^{2}=\left\|U\left(x^{2}\right)\right\|=\inf \left\{\left\|x^{2}+p\right\|: p \in P\right\}$, by 2.2 (c), =inf $\left\{\left\|x^{2}+y^{2}\right\|: y \in R\right\}=\left\|x^{2}\right\|=\|x\|^{2}$, the last two equalities following directly from our hypotheses on $R$. Thus $U$ is an isometry. That $U$ is an isomorphism follows at once, and, by 2.2 , the image of $R$ is dense, and being now complete, is therefore all of $R(X)$.

\section{3. * Algebras.}

3.1. Definitions. A * algebra $A$ is a complex Banach algebra with unit 1 of norm 1 and with a unary operation ${ }^{*}$ such that $x^{* *}=x,\|x\|=\left\|x^{*}\right\|$, $(x y)^{*}=y^{*} x^{*}$ and $(a x+b y)^{*}=\bar{a} x^{*}+b y^{*}$ for all $x, y \in A$ and all complex numbers $a, b$. The set $H$ of hermitean or self-adjoint elements is $\left\{x: x=x^{*}\right\}$ and the positive cone $P$ is the closure of the set of all finite sums of elements of the form $x x^{*}$. (Note that $H$ is a real Banach space and $P$ is a cone in $H$.) The set $P^{\prime}$ of functionals of positive type is the set of all $f \in A^{*}$ such that $f\left(x x^{*}\right) \geqq 0$ for $x \in A$. The intersection of $P^{\prime}$ with the unit sphere in $A^{*}$ is $\Sigma$.

3.2. Remarks. Each $x \in A$ is uniquely expressible in the form $u+i v$ where $u, v \in H$. (Directly: $u=\left(x+x^{*}\right) / 2$ and $v=\left(x-x^{*}\right) / 2 i$.) If $f \in H^{*}$ and we set $F(u+i v)=f(u)+i f(v)$, then $F \in A^{*}$ and $F\left(x^{*}\right)=\bar{F}(x)$. Conversely, if $F \in A^{*}$ and $F\left(x^{*}\right)=\bar{F}(x)$ for $x \in A$, then $F$ is real on $H$. If $F\left(x x^{*}\right) \geqq 0$ for each $x \in A$, then $F\left(x^{*}\right)=\bar{F}(x)$. (Consider $F\left(\left(e^{-i \theta}+x\right)\left(e^{i \theta}+x^{*}\right)\right)$.) Thus $H^{*}$ is embedded naturally in $A^{*}$, so that members of the dual cone of $P$, i.e., members of $H^{*}$ which are non-negative on $P$, correspond exactly to members of $P^{\prime}$. If $A$ is not commutative, $H$ may not be a subalgebra. However, powers of hermitean elements are hermitean, and if $h \in H$ and $\|h\| \leqq 1$, then, by the same power series argument, $1-h$ is the square of an hermitean element. Consequently $P$ contains the unit sphere in $H$ about 1 and Lemma 1.3 is applicable. Finally, 
we remark that if $F \in P^{\prime},\|F\|=F(1)$, and thus the norms of $F$ and its restriction to $H$ are identical. In fact, $F\left(y^{*} x\right)$ defines an inner product on $A$ and, by the Cauchy-Schwartz inequality and then Lemma 1.3, $|F(x)|^{2} \leqq F\left(x^{*} x\right) F(1)$ $\leqq F(1)\left\|x^{*} x\right\| F(1) \leqq F(1)^{2}\|x\|^{2}$. Thus Lemma 1.3 now gives us:

\subsection{Lemma. For $x=x^{*} \in A$, dist $(-x, P)=\sup \left\{f(x): f \in P^{\prime}\right.$ and $\left.\|f\| \leqq 1\right\}$.}

In the remainder of $\$ 3$ we shall suppose $A$ is commutative. In this case $H$ is a real algebra, and it is easy to check that the set of real homomorphisms of $H$ is in the natural correspondence with the set $Y_{0}$ of homomorphisms $h$ of $A$ into the complex numbers for which $h\left(x^{*}\right)=\bar{h}(x)$ for $x \in A$. Such homomorphisms are of real type. (In general, there may be homomorphisms of $A$ into the complex numbers which are not of real type. It is easy to see that all such homomorphisms are of real type if and only if $A$ is symmetric in the sense of Gelfand and Naimark [2], i.e., if and only if $1+x x^{*}$ has an inverse for each $x \in A$.) The results of $\$ 2$ make the proof of the following theorem easy.

3.4. Theorem. Let $A$ be a commutative * algebra and let $U$ be the natural *-preserving homomorphism of $A$ into the * algebra $C(Y)$ of all complex-valued continuous functions on the (compact) space $Y$ of nontrivial homomorphisms of $A$ of real type, with the $w^{*}$ topology. Then $\left({ }^{5}\right)$ :

(a) The image of $A$ under $U$ is dense in $C(Y)$,

(b) For $x=x^{*} \in A$, dist $(-x, P)=\max [0, \sup \{f(x): f \in Y\}]$,

(c) For $x \in A,\|U(x)\|^{2}=\sup \left\{f\left(x x^{*}\right): f \in Y\right\}=\operatorname{dist}\left(-x x^{*}, P\right)$,

(d) ( $\left.{ }^{6}\right)$ If $\left\|x x^{*}\right\|=\|x\|^{2}$ for each $x \in A$, then $U$ is an isometry (and hence an isomorphism of $A$ onto $C(Y))$.

Proof. (a) follows from the Stone-Weierstrass theorem, (b) from Theorem 2.2, and (c) from (b) and the fact that $\|U(x)\|^{2}=\|U(x)\|\|\bar{U}(x)\|=\left\|U\left(x x^{*}\right)\right\|$. To prove (d): for $x, y \in H, 2 x=x+i y+x-i y$, so that $2\|x\| \leqq\|x+i y\|+\|x-i y\|$ $=2\|x+i y\|$; consequently $\left\|x^{2}+y^{2}\right\|=\|(x+i y)(x+i y) *\|=\|x+i y\|^{2} \geqq\|x\|^{2}$. By 2.4 and the remarks ending 3.2 , it follows that $\|U(x)\|=\|x\|$ for $x \in H$. Hence for any $x \in A,\|x\|^{2}=\left\|x x^{*}\right\|=\left\|U\left(x x^{*}\right)\right\|=\|U(x)\|^{2}$, which completes the proof.

3.5. Remarks. Calling the kernel $K$ of the homomorphism $U$ the ${ }^{*}$ radical of $A$, we see that, for any $x \in A$, the following conditions are equivalent: (a) $x \in K$, (b) $-x x^{*} \in P$, (c) $h(x)=0$ for every homomorphism of real type.

4. Noncommutative * algebras.

4.1. Preliminaries. If $A$ is not commutative, the algebraic significance of the positive functionals is more complicated. We shall first outline briefly the construction due to Gelfand and Naimark [3] (see also Segal [11]).

(8) This result is due to Gelfand and Naimark [2]. See also Arens [1]. It is to be remarked that in Arens' work, the representation of commutative ${ }^{*}$ algebras is obtained first and then used to obtain the representation theorem for real Banach algebras, in contrast to our development. 
An operator representation $U$ of $A$ is an algebraic homomorphism of $A$ into the * algebra of all bounded operators on a Hilbert space. (In particular, $U_{x^{*}}$ is the operator adjoint to $U_{x}$.) We include the one element Hilbert space, for convenience. It follows that $U$ is bounded, and in fact $\|U\|=\left\|U_{1}\right\|$ $=\left\|U_{1}\right\|^{2}=1$ or 0 . To see this, let $\|x\| \leqq 1$. Then $\left\|x x^{*}\right\| \leqq 1$, so $1-x x^{*}$ is the square of an hermitean element. Thus $U_{1}, U_{x x^{*}}$, and $U_{1}-U_{x x^{*}}$ are positive operators, and so $\left\|U_{x}\right\|^{2}=\left\|U_{x x^{*}}\right\| \leqq\left\|U_{1}\right\|=\left\|U_{1}\right\|^{2}$.

Corresponding to each $f \in P^{\prime}$ we construct an operator representation $U^{f}$ as follows: Let $(x, y)_{f}=f\left(y^{*} x\right)$ for $x, y \in A$. Then $(,)_{f}$ is an inner product on $A$ and the quotient $A$ modulo $I_{f}=\left\{x: f\left(x^{*} x\right)=0\right\}$ is a possibly incomplete Hilbert space which can be embedded in a complete Hilbert space $\mathfrak{K}_{f}$. For fixed $y$, let $g(z)=f\left(y^{*} z y\right)$ and observe that $g \in P^{\prime}$, so that $\|g\|=g(1)$, and hence $g\left(x^{*} x\right) \leqq g(1)\left\|x^{*} x\right\| \leqq g(1)\|x\|^{2}$, i.e., $f\left(y^{*} x^{*} x y\right) \leqq f\left(y^{*} y\right)\|x\|^{2}$. Using this relation, we see that $I_{f}$ is a left ideal, and that for $x \in A$ we may define $U_{x}^{f}$ as the unique extension to all of $\mathfrak{F C}_{f}$ of the (bounded) operator such that $U_{x}^{f}\left(y / I_{f}\right)=(x y) / I_{f}$ for $y \in A$. It is easy to check that $U^{f}$ is an operator representation of $A$.

Now let $\mathfrak{H}$ be the Hilbert space product $\prod\left\{\mathfrak{C}_{f}: f \in P^{\prime}\right\}$, and let $V$ be defined coordinate-wise by setting $\left(\mathcal{V}_{x} \xi\right)_{f}=U_{x}^{\prime} \xi_{f}$ for $x \in A, \xi \in \mathcal{H}$, and $f \in P^{\prime}$. Then $V$ is an operator representation of $A$, which, as we shall see, is in a sense a universal operator representation of $A$.

In the following, the upper bound of an hermitean operator $B$ is defined, for convenience, as $u b_{0} B=\sup \{(B \xi, \xi):\|\xi\| \leqq 1\}$. Note that this differs from the usual upper bound, and is, in fact, equal to the greater of the usual upper bound and 0 .

4.2. Lemma. Let $x=x^{*} \in A$. Then $u b_{0} \mho_{x}=\sup \left\{u b_{0} U_{x}^{p}: f \in P^{\prime}\right\}=\sup$ $\left\{u b_{0} U_{x}: U\right.$ any operator representation of $\left.A\right\}$.

Proof. Let $a, b, c$ be, in order, the three numbers asserted to be equal. That $a \geqq b$ follows in straightforward manner from the definitions of product space and upper bound and that $c \geqq a$ is obvious. It remains to prove that $b \geqq c$. Let $U$ be any operator representation over a Hilbert space, and $\xi$ any member of that Hilbert space with $\|\xi\| \leqq 1$. Set $f(y)=\left(U_{y} \xi, \xi\right)$ for $y \in A$. Then $f \in P^{\prime}$, and $\left\|1 / I_{f}\right\|_{f}^{2}=f(1)=\left(U_{1} \xi, \quad \xi\right)=\left(U_{1} \xi, \quad U_{1} \xi\right)=\left\|U_{1} \xi\right\|^{2} \leqq\|\xi\|^{2} \leqq 1$, and $\left(U_{x}^{f}\left(1 / I_{f}\right), 1 / I_{f}\right)_{f}=f(x)=\left(U_{x} \xi, \xi\right)$, which proves that $b \geqq c$.

This last argument can be extended to show that any representation $U$ in which, for some $\xi,\left\{U_{x} \xi: x \in A\right\}$ is dense in the Hilbert space is unitarily equivalent to one of the $U^{f}$, and, more generally, that any operator representation is unitarily equivalent to a product of representations $U^{f}$. (Cf. [3].)

4.3. Lemma. Let $x=x^{*} \in A$. Then sup $\left\{g\left(y^{*} x y\right): y \in A, g\left(y^{*} y\right) \leqq 1\right.$, and $\left.g \in P^{\prime}\right\}=\sup \left\{f(x): f \in P^{\prime}\right.$ and $\left.f(1) \leqq 1\right\}$.

Proof. For $g \in P^{\prime}$ and $y$ such that $g\left(y^{*} y\right) \leqq 1$, let $f(z)=g\left(y^{*} z y\right)$ for $z \in A$. 
Then $f(1) \leqq 1$, and $f \in P^{\prime}$, and $f(x)=g\left(y^{*} x y\right)$. Thus the second member is at least as great as the first. The reverse inequality can be obtained by setting $y=1$ in the first member.

4.4. Theorem. Let $A$ be $a{ }^{*}$ algebra, $P$ its positive cone, $P^{\prime}$ the set of positive functionals, $U^{f}$ the operator representation corresponding to a positive functional $f$, and $U$ the product of all the representations $U^{j}$. Then:

(a) for $x=x^{*} \in A$,

$$
\begin{aligned}
\operatorname{dist}(-x, P) & =\sup \left\{u b_{0} U_{x}^{f}: f \in P^{\prime}\right\} \\
& =\sup \left\{u b_{0} U_{x}: U \text { an operator representation of } A\right\} \\
& =u b_{0} U_{x}
\end{aligned}
$$

(b) for $x \in A$,

$$
\begin{aligned}
\operatorname{dist}\left(-x x^{*}, P\right) & =\sup \left\{\left\|U_{x}\right\|^{2}: U \text { an operator representation of } A\right\} \\
& =\left\|U_{x}\right\|^{2} .
\end{aligned}
$$

Proof. (a) follows from Lemmas 3.3, 4.3, and 4.2, and the fact that $u b_{0} U_{x}^{f}=\sup \left\{f\left(y^{*} x y\right): f\left(y^{*} y\right) \leqq 1\right\}$. (b) follows from (a), since, for any representation $U, u b_{0} U_{x x^{*}}=u b_{0} U_{x} U_{x^{*}}=\left\|U_{x} U_{x^{*}}\right\|=\left\|U_{x}\right\|^{2}$.

4.5. Remarks. We shall apply 4.4 to three problems, namely, to obtain necessary and sufficient conditions for the existence of an operator representation of $A$ which is also (a) an isomorphism, (b) a bicontinuous isomorphism, (c) an isometry. From the fact that $\left\|V_{x}\right\|=\sup \left\{\left\|U_{x}\right\|: U\right.$ an operator representation of $A\}$, it follows that it is equivalent to ask whether $U$ is one of (a), (b), (c), respectively.

The ${ }^{*}$ radical of $A$ is defined to be the kernel of $V$, i.e., $\left\{x: U_{x}=0\right.$ for all operator representations $U\}$. From 4.4, we see that if $x=x^{*}$, the following are equivalent: (a) $x \in K$, (b) $x \in P$ and $-x \in P$, (c) $f(x)=0$ for all $f \in P^{\prime}$. By breaking into real and imaginary parts, it follows that for any $x \in A$, the following are equivalent: (a) $x \in K$, (b) $-x x^{*} \in P$, (c) $x^{*} x \in K$, (d) $f\left(x^{*} x\right)=0$ for all $f \in P^{\prime}$, (e) $f(x)=0$ for all $f \in P^{\prime}$. (Cf. Raikov [9].) Thus $K=(P \cap-P)$ $+i(P \cap-P)$; and the * radical is $\{0\}$, i.e., $U$ is an isomorphism if and only if $P \cap-P=\{0\}$.

(It is well known that if $f \in P^{\prime}$ and $f(1)=1$, then $U^{f}$ is irreducible if and only if $f$ is an extreme point of $\Sigma$. (See, e.g., [3].) Applying Lemma 1.6 then shows that $\left\|V_{x}\right\|$ is also equal to the supremum of the norms $\left\|U_{x}\right\|$ for $U$ an irreducible operator representation. These facts, with the fact that an irreducible representation of an Abelian * algebra is one-dimensional, enable the results on Abelian * algebras to appear as special cases of the present results, and make clear the consistency of our two definitions of the ${ }^{*}$ radical.)

4.6. TheOREM. $U$ is a bicontinuous isomorphism if and only if $\{x: 1+x$, $1-x \in P\}$ is bounded. 
Proof. Let $x=x^{*}$. Since dist $(x, P)=u b_{0}-U_{x}, x \in P$ if and only if $V_{x}$ is a positive operator. Recall that an hermitean operator $B$ has norm $\leqq 1$ if and only if both $1-B$ and $1+B$ are positive, and note that $V_{1}=1$. Thus the set of hermitean elements of $A$ mapping into the unit sphere of the operator algebra under $V$ is precisely $\{x: 1+x, 1-x \in P\}$. It is easy to see that this set is bounded if and only if the entire inverse image under $V$ of the unit sphere of the operator algebra is bounded, and the latter is a necessary and sufficient condition that a continuous linear map be bicontinuous.

Note that if $V$ is a bicontinuous isomorphism, the image of $A$ is complete, and is therefore a $C^{*}$ algebra in the terminology of [11], i.e., a * algebra of operators on a Hilbert space, closed in the uniform or norm topology.

We now consider the problem of characterizing those ${ }^{*}$ algebras which are isomorphic and isometric to a $C^{*}$ algebra, i.e., for which $U$ is an isometry. We do not succeed in proving the conjecture of Gelfand and Naimark [2] that $\left\|x x^{*}\right\|=\|x\|^{2}$ is a sufficient condition. However, Theorem 4.7 below may be of interest in itself. (The same argument used in its proof suffices to prove the closely related result of Sherman [13] that, in the terminology of Segal [12], non-negative observables are squares.)

4.7. Theorem. Let $A$ be a ${ }^{*}$ algebra such that $\left\|x x^{*}\right\|=\|x\|^{2}$ for each $x \in A$, and let $Q$ be the set of all squares of hermitean elements. Then:

(a) $Q=\left\{x: x=x^{*}\right.$ and either $x=0$ or $\left.\|1-x /\| x\|\| \leqq 1\right\}$.

(b) $Q$ is a cone in $H$.

(c) For $x$ hermitean, dist $\left(-x^{2}, Q\right)=\left\|x^{2}\right\|$.

(d) For $x, y$ hermitean, $\left\|x^{2}+y^{2}\right\| \geqq\left\|x^{2}\right\|$.

(e) If also $x x^{*}$ is the square of an hermitean element for each $x \in A$, then $v$ is an isometry.

Proof. Recall that if $x \in H$ and $\|1-x\| \leqq 1$, then $x \in Q$. We now have the partial converse: if $x \in Q$ and $\|x\| \leqq 1$, then $\|1-x\| \leqq 1$. In fact, let $x=u^{2}$, $u \in H,\|x\| \leqq 1$, and let $A(u)$ be the commutative closed * subalgebra generated by $u$ and 1 . By $3.4(\mathrm{~d}), A(u)$ is isometric to a function algebra, and so, since $\left\|u^{2}\right\| \leqq 1$, it is clear that $\left\|1-u^{2}\right\| \leqq 1$.

(a) follows at once, and from (a) we see that $Q$ is closed. We complete the proof that $Q$ is a cone by showing that if $x, y \in Q$, then $x+y \in Q$. We may presume that $\|x\|,\|y\| \leqq 1$. Then $\|1-x\|,\|1-y\| \leqq 1$, and so $\|1-(x+y) / 2\|$ $\leqq 1$, and hence $(x+y) / 2, x+y \in Q$.

To prove (c), let $r=\operatorname{dist}\left(-x^{2}, Q\right)$ and $A(x)$ as above. The cone $Q$ in $H$ satisfies the hypotheses of Lemma 1.3, and hence $r \cdot 1-x^{2} \in Q$. In view of the characterization of $Q$ in (a), applied now relative to $A(x), r \cdot 1-x^{2}$ is also the square of an hermitean element $z$ of $A(x)$. Thus in the "function algebra" $A(x), r \cdot 1=x^{2}+z^{2}$, and so dist $\left(-x^{2}, Q\right)=r=\left\|x^{2}+z^{2}\right\| \geqq\left\|x^{2}\right\|$. This proves (c), and (d) follows at once from (c).

Finally, under the additional hypothesis of (e), any $x x^{*} \in Q$, and since $Q$ 
is a cone, it follows that $P=Q$. Hence, by 4.4 and (c), for any $x \in A,\left\|\mathcal{V}_{x}\right\|^{2}$ $=\operatorname{dist}\left(-x x^{*}, P\right)=\operatorname{dist}\left(-x x^{*}, Q\right)=\left\|x x^{*}\right\|=\|x\|^{2}$, so that $U$ is an isometry.

Various other criteria for a $C^{*}$ algebra follow rather easily from 4.7 (e). We shall for completeness include the theorem of Gelfand and Naimark $[2]\left({ }^{7}\right)$.

4.8. TheOREM. In order that $a *$ algebra $A$ be isomorphic and isometric to a $C^{*}$ algebra, it is necessary and sufficient that $\left\|x x^{*}\right\|=\|x\|^{2}$ and that $1+x x^{*}$ have an inverse, for each $x \in A$.

Proof. We need only show that under the hypotheses, every $x x^{*}$ is the square of an hermitean element. For $x=x^{*}$, let $A(x)$ be as before. If $x=x^{*}$ and $\lambda \cdot 1-x$ has an inverse, the smallest closed * subalgebra containing $\lambda \cdot 1-x,(\lambda \cdot 1-x)^{-1}$, and 1 is clearly commutative and, by $3.4(\mathrm{~d})$ and the Stone-Weierstrass theorem, is identical with $A(\lambda \cdot 1-x)$ and hence with $A(x)$. Thus the spectrum of an hermitean element $x$ is the same relative to $A$ and to $A(x)$. Under our assumption, for any $x \in A, x x^{*}$ has a non-negative spectrum, and so, considering the "function algebra" $A\left(x x^{*}\right)$, is the square of an hermitean element.

\section{Application to groups.}

5.1. Preliminaries. Let $G$ be a locally compact Abelian topological group. A Radon measure (or simply, measure) on $G$ is a finite linear combination, with complex coefficients, of outer regular measures on the Baire $\sigma$-ring of $G$. (Cf. [4].) The set $B$ of Radon measures is in one to one linear correspondence with the adjoint of $C_{\infty}(G)$, where $C_{\infty}(G)$ is the Banach space of all complex continuous functions on $G$ which vanish at infinity, with the supremum norm. We norm $B$ with the norm of $\left(C_{\infty}(G)\right)^{*}$. For $m, n \in B, m n$ is the convolution, i.e., $\int f(x) d m n x=\iint f(x y) d m x d n y$ for $f \in C_{\infty}(G)$, while $m^{*}$ is defined by the requirement that $\int f(x) d m^{*} x=\left[\int \bar{f}\left(x^{-1}\right) d m x\right]-$ for $f \in C_{\infty}(G)$, where the bar indicates complex conjugate. $B$ is then $a^{*}$ algebra. For $a \in G$, the point measure at $a$ is the measure $m$ such that $\int f(x) d m x=f(a)$ for $f \in C_{\infty}(G)$. A measure $m$ is discrete if it is a countable linear combination of point measures.

Let $L$ be the set of all measures absolutely continuous with respect to Haar measure. $L$ is a closed * ideal in $B$, and is isomorphic and isometric to the usual $L_{1}$ algebra of the group. Let $A$ be the set of all measures $m=m_{1}+m_{2}$, where $m_{1}$ is discrete and $m_{2} \in L$. It is easy to see that $\left\|m_{1}+m_{2}\right\|=\left\|m_{1}\right\|+\left\|m_{2}\right\|$, and hence that $A$ is itself a ${ }^{*}$ algebra, and the representation $m=m_{1}+m_{2}$, as above, is unique (unless $G$ is discrete, in which case all measures in $B$ are discrete). Theorem 3.4(b) tells us that if $P$ is the positive cone of $A$ and $m=m^{*} \in A$, then $\operatorname{dist}(-m, P)=\max [0, \sup \{f(m): f$ a nontrivial homomorphism of $A$ of real type $\} \cdot$. Using techniques due to Segal [10], this can be refined so as to give us a bound for the Fourier transform of a measure in $A$.

(7) See Kaplansky [5] for a formulation and proof of this result in the case where $A$ has no unit element. 
Let $G^{\prime}$ be the group $G$ considered as a discrete group, and $G^{*}$ be the continuous character group of $G$. For $m \in A, f \in G^{*}$, let $F_{m}(f)=\int f(t) d m t$. The continuous function $F_{m}$ on $G^{*}$ is the Fourier transform of $m$. Similarly we define $G^{*}$ and $F^{\prime}$. The complex homomorphisms of $A$ are of two sorts $\left.{ }^{8}\right)$. If $f \in G^{*}$ and $h(m)=F_{m_{1}}(f)$ for $m=m_{1}+m_{2} \in A$, or if $g \in G^{*}$ and $h(m)=F_{m}(g)$ for $m \in A$ then $h$ is a homomorphism of $A$ of real type. Conversely, suppose $h$ is any complex homomorphism of $A$. If $h$ vanishes on $L$, it is easy to see that $h$ is of the first type. Otherwise $h(n)=1$ for some $n \in L$, and for some $f \in G^{*}$ we have $h(m)=\int f(t) d m t$ for $m \in L[10$, Theorem 1.9, p. 80]. Thus for any $m \in A$, since $m n \in L$, we have $h(m)=h(m n)=\int f(t) d m n t=\iint f(s t) d m s d n t$ $=\int f(s) d m s \int f(t) d n t=F_{m}(f) h(n)=F_{m}(f)$, and $h$ is of the second type. In particular, we see that all complex homomorphisms of $A$ are of real type.

5.2. Lemma. If $G$ is not discrete and $m=m^{*} \in A$, then $\sup \left\{F_{m}(f): f \in G^{*}\right\}$ $\geqq \sup \left\{F_{m_{1}}^{\prime}(f): f \in G^{*}\right\}$.

Proof. It is well known that $G^{*}$ is a dense subgroup of $G^{*}$; hence we may replace the right member of the inequality by sup $\left\{F_{m_{1}}(f): f \in G^{*}\right\}$. Since $m_{1}$ is discrete and $m_{2}$ absolutely continuous, $F_{m_{1}}$ is almost periodic, and $F_{m_{2}}$ vanishes at infinity in $G^{*}[16$, p. 116]. Let $\epsilon>0$. We may choose $C$ compact in $G^{*}$ so that $\left|F_{m_{2}}(f)\right|<\epsilon$ when $f \in G^{*}-C$ and thus sup $\left\{F_{m_{1}}(f): f \in G^{*}-C\right\}$ $\leqq \sup \left\{F_{m}(f): f \in G^{*}\right\}+\epsilon$. An obvious modification of a lemma of Segal [10, Lemma 3.7.1. p. 99] tells us that

$$
\sup \left\{F_{m_{1}}(f): f \in G^{*}-C\right\}=\sup \left\{F_{m_{1}}(f): f \in G^{*}\right\},
$$

since $F_{m_{1}}$ is almost periodic. Thus $\sup \left\{F_{m_{1}}(f): f \in G^{*}\right\} \leqq \sup \left\{F_{m}(f): f \in G^{*}\right\}+\epsilon$, and the lemma is proved.

5.3. Theorem. If $G$ is a locally compact Abelian group, $A$ the algebra of measures each of which is the sum of a discrete measure and a measure absolutely continuous with respect to Haar measure, and $F_{m}$ is the Fourier transform of $m \in A$, then:

(a) $\sup \left\{\left|F_{m}(f)\right|^{2}: f \in G^{*}\right\}=\inf \left\{\left\|m m^{*}+n^{2}\right\|: n \in A\right.$ and $\left.n=n^{*}\right\}$,

(b) if $m=m^{*}$, then

$$
\max \left[0, \sup \left\{F_{m}(f): f \in G^{*}\right\}\right]=\inf \left\{\left\|m+n^{2}\right\|: n \in A \text { and } n=n^{*}\right\} \text {. }
$$

Proof. If $P$ is the positive cone of $A, m \in P$, and $k>0$, then, since all complex homomorphisms of the commutative * algebra $A$ are of real type, it is clear that the spectrum of $m+k \cdot 1$ is strictly positive. Raikov [9, p. 389] has shown by contour integration that $m+k \cdot 1$ is therefore the square of an hermitean element of $A$. Such squares are therefore dense in $P$, and

(8) Unless $G$ is discrete, in which case it is easy to see that for any complex homomorphism $h$, there is a character $f$ with $F_{m}(f)=h(m)$ and $m \in A$. In the remainder of this paragraph, assume $G$ is not discrete. 
$\operatorname{dist}(-m, P)=\inf \left\{\left\|m+n^{2}\right\|: n \in A\right.$ and $\left.n=n^{*}\right\}$ for any $m \in A$. (b) then follows from Theorem 3.4(b), our identification of the complex homomorphisms of $A$, and Lemma 5.2. (a) follows from (b), since $\left|F_{m}(f)\right|^{2}=F_{m m^{*}}(f)$.

5.4. Remarks. Theorem 5.3, applied to the group of integers, gives expressions for the upper bound of a real Fourier series and the upper bound of the absolute value of any Fourier series which seem to be new. Using Fejér's theorem on positive trigonometric polynomials (see, for example, $[15$, p. 3]), it is not difficult to see that here measures of the form $m^{*} m$, where $m$ consists of masses placed at a finite number of non-negative integers, are dense in $P$. Thus, for example, if $f(t)=\sum_{n=-\infty}^{\infty} a_{n} e^{i n t}$ is absolutely convergent, and is realvalued (i.e., $a_{n}=\bar{a}_{-n}$ ), and if $S$ is the class of all (two-ended) sequences which are 0 except for a finite number of terms with non-negative indices, then $\max [0, \sup \{f(t): 0 \leqq t<2 \pi\}]=\inf \left\{\sum_{n=-\infty}^{\infty}\left|a_{n}+\sum_{m=-\infty}^{\infty} b_{m} \bar{b}_{m-n}\right|: b \in S\right\}$.

\section{BiBLIOGRAPHY}

1. Richard Arens, Representations of *-algebras, Duke Math. J. vol. 14 (1947) pp. 269-282.

2. I. Gelfand and M. Naimark, On the imbedding of normed rings into the ring of operators in Hilbert space, Rec. Math. (Mat. Sbornik) N.S. vol. 12 (1943) pp. 197-213.

3. - Normed rings with involutions and their representations, Izvestiya Akademii Nauk SSSR. Ser. Mat. vol. 12 (1948) pp. 445-480.

4. P. R. Halmos, Measure theory, New York, 1950.

5. R. V. Kadison, A representation theory for commutative topological algebra, Memoirs of the American Mathematical Society, no. 7, 1951.

6. I. Kaplansky, Normed algebras, Duke Math. J. vol. 16 (1949) pp. 399-418.

7. M. Krein and D. Milman, On extreme points of regular convex sets, Studia Mathematica vol. 9 (1940) pp. 133-137.

8. M. Krein and M. Rutman, Linear operators leaving invariant a cone in a Banach space, Uspehi Matematǐeskih Nauk. vol. 3 (23) (1948) pp. 3-95.

9. D. A. Raikov, To the theory of normed rings with involutions, C. R. (Doklady) Acad. Sci. URSS. vol. 54 (1946) pp. 387-390.

10. I. E. Segal, The group algebra of a locally compact group, Trans. Amer. Math. Soc. vol. 61 (1947) pp. 69-105.

11. - Irreducible representations of operator algebras, Bull. Amer. Math. Soc. vol. 53 (1947) pp. 73-88.

12. - Postulates for general quantum mechanics, Ann. of Math. vol. 48 (1947) pp. 930-948.

13. S. Sherman, Non-negative observables are squares, Proceedings of the American Mathematical Society vol. 2 (1951) pp. 31-33.

14. M. H. Stone, Applications of the theory of Boolean rings to general topology, Trans. Amer. Math. Soc. vol. 41 (1937) pp. 375-481.

15. G. Szegö, Orthogonal polynomials, Amer. Math. Soc. Colloquium Publications, vol. 23, New York, 1939.

16. A. Weil, L'intégration dans les groupes topologiques et ses applications, Actualités Scientifiques et Industrielles, no. 869, Paris, 1940.

Tulane University, New OrLeans, La.

UNIVERSITY OF CaLIForNia, Berkeley, Calif. 